SCIENTIFIC LETTER

\title{
Progression of native coronary plaques and in-stent restenosis are associated and predicted by increased pre-procedural $C$ reactive protein
}

\author{
D Skowasch, A Jabs, R Andrié, B Lüderitz, G Bauriedel
}

Heart 2005;91:535-536. doi: 10.1136/hrt.2004.037317

$\mathrm{b}$ n-stent restenosis (ISR) is a major limitation of coronary bare metal stent implantation. While investigational work in the field has mainly focused on the formation as well as putative predictors of ISR, little is known about the simultaneous progression of primarily untreated plaques in the coronary tree within the same patient. Without any interventional therapy, progression has been reported to occur in $7-20 \%$ of coronary plaques per year. Also, plaque progression apparently increases with severity of coronary artery disease as well as with patient anginal status. ${ }^{1}$

In the present prospective study, we evaluated a patient cohort undergoing coronary stent implantation and six month re-angiogram to test whether progression in primarily untreated plaques would occur more frequently with target lesion ISR.

\section{METHODS}

In a prospective observational single centre registry, symptomatic patients undergoing first successful single stent implantation were enrolled between May 2001 and April 2002 at the Department of Cardiology, University of Bonn, Germany. Exclusion criteria were: angioplasty without stenting; presence of restenosis in the initial angiogram or previous coronary artery bypass grafting; multiple stent implantation in one lesion, or stenting in more than one lesion; significant left main coronary artery lesion; three vessel disease or chronic total occlusions; infection or inflammatory disease; and lack of angiographic follow up. Of 274 patients with first single stent implantation in native coronary lesions, control angiograms were done in 219 patients ( $80 \%$ of the eligible patients) at $>3$ months and $<9$ months, mean time 6 (1) months. In all patients, serum was collected before stent implantation, and $\mathrm{C}$ reactive protein (CRP) concentrations were measured using a commercially available kit ( $\mathrm{N}$ High Sensitivity CRP, Dade Behring, Marburg, Germany).

Coronary angiograms were analysed by two independent observers and were evaluated with the Coronary Angiography Analysis System (CAAS II for Windows QCA V4.1, Pie Medical Imaging BV, Maastricht, The Netherlands). Baseline stenoses of $>20 \%$, as compared to the proximal reference segment, were assessed for progression/regression, defined as $>20 \%$ increase/decrease in diameter stenosis. Any novel localised narrowing of $>30 \%$ at follow up was regarded as development of a de novo lesion. ISR was defined as $>50 \%$ diameter stenosis at follow up. Categorical variables were assessed by $\chi^{2}$ analysis. Normally distributed continuous data are presented as mean (SD). For univariate analyses comparisons between groups were made using the unpaired Student $t$ test; probability values of $\mathrm{p}<0.05$ were considered significant.

\section{RESULTS}

Serial quantitative coronary analysis was completed in 219 patients treated by stent implantation for symptomatic coronary artery disease. A total of 219 stented segments and 134 untreated plaques were evaluated at the time of stent deployment and at follow up angiogram after 6 (1) months.

ISR was found in 61 of $219(28 \%)$ target lesions. The ISR and non-ISR subgroups did not differ with respect to clinical variables such as age, sex, and medication (table 1). Only diabetes mellitus was more prevalent with ISR $(p<0.001)$, while other demographic and patient characteristics were comparable within both groups. Notably, pre-procedural CRP concentrations were substantially increased $(p=0.02)$ in patients with subsequent ISR (11.0 (16.0) mg/l $v 5.6$ (11.6) $\mathrm{mg} / \mathrm{l}$ ). Also, ISR development was associated with length of target lesion $(\mathrm{p}=0.02)$, while other angiographic and procedural variables did not significantly differ between groups.

A total of 15 of 134 (11\%) untreated coronary plaques in 15 of $219(7 \%)$ patients demonstrated angiographic progression (fig 1). Significant lesion developments within an initially

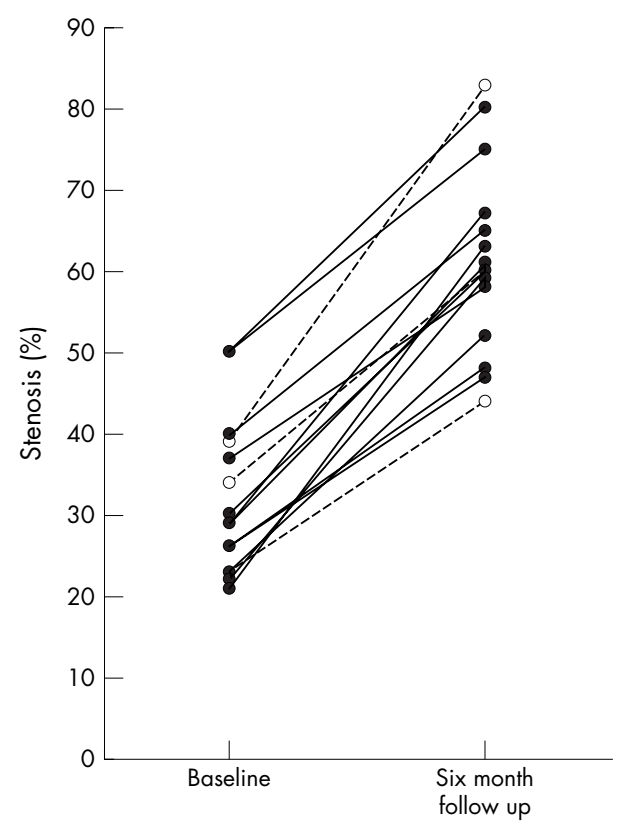

Figure 1 Progression of untreated coronary lesions from baseline (left) to follow up (right). Mean diameter stenosis of the 15 progressive plaques was $32 \%$ at baseline and increased to an average value of $61 \%$ at 6 month follow up. Solid lines indicate an association with in-stent restenosis (ISR) formation; broken lines mark progression without ISR. 
Table 1 Baseline characteristics of patient collective with regard to ISR development within the lesion and progression of other untreated plaques

\begin{tabular}{llllll}
\hline & All & ISR & No ISR & Progress & No progress \\
\hline Number of patients (\%) & $219(100 \%)$ & $61(28 \%)$ & $158(72 \%)$ & $15(7 \%)$ & $204(93 \%)$ \\
Age (years) & $63(10)$ & $63(11)$ & $64(10)$ & $63(9)$ & $63(10)$ \\
Male sex & $173(79 \%)$ & $48(78 \%)$ & $126(80 \%)$ & $13(87 \%)$ & $157(77 \%)$ \\
ACS & $118(54 \%)$ & $39(64 \%)$ & $71(45 \%)$ & $9(60 \%)$ & $108(53 \%)$ \\
Acute MI & $53(24 \%)$ & $16(26 \%)$ & $35(22 \%)$ & $4(27 \%)$ & $47(23 \%)$ \\
Previous MI & $61(28 \%)$ & $19(31 \%)$ & $41(26 \%)$ & $3(20 \%)$ & $59(29 \%)$ \\
Risk factors & & & & & \\
Diabetes & $42(19 \%)$ & $17(28 \%)^{*}$ & $17(11 \%)^{*}$ & $3(20 \%)$ & $37(18 \%)$ \\
Hypertension & $136(62 \%)$ & $41(67 \%)$ & $92(58 \%)$ & $10(67 \%)$ & $126(62 \%)$ \\
Hypercholesterolaemia & $83(38 \%)$ & $27(44 \%)$ & $51(32 \%)$ & $8(53 \%)$ & $73(36 \%)$ \\
Current smoker & $94(43 \%)$ & $30(49 \%)$ & $60(38 \%)$ & $12(80 \%)^{* *}$ & $80(39 \%)^{* *}$ \\
Family history of CAD & $68(31 \%)$ & $21(34 \%)$ & $43(27 \%)$ & $4(27 \%)$ & $63(31 \%)$ \\
Medication & & & & & \\
Aspirin & $219(100 \%)$ & $61(100 \%)$ & $158(100 \%)$ & $15(100 \%)$ & $204(100 \%)$ \\
Clopidogrel & $219(100 \%)$ & $61(100 \%)$ & $158(100 \%)$ & $15(100 \%)$ & $204(100 \%)$ \\
Nitrate & $96(44 \%)$ & $24(39 \%)$ & $76(48 \%)$ & $5(33 \%)$ & $92(45 \%)$ \\
B Blocker & $99(45 \%)$ & $34(56 \%)$ & $57(36 \%)$ & $10(67 \%)$ & $96(47 \%)$ \\
ACE inhibitor & $99(45 \%)$ & $35(57 \%)$ & $54(34 \%)$ & $8(53 \%)$ & $106(52 \%)$ \\
ATI antagonist & $18(8 \%)$ & $4(7 \%)$ & $16(10 \%)$ & $1(7 \%)$ & $16(8 \%)$ \\
Calcium antagonist & $28(13 \%)$ & $13(21 \%)$ & $11(7 \%)$ & $1(7 \%)$ & $29(14 \%)$ \\
Statin & $99(45 \%)$ & $35(57 \%)$ & $55(35 \%)$ & $10(67 \%)$ & $102(50 \%)$ \\
GP Ilb/Illa antagonist & $55(25 \%)$ & $15(25 \%)$ & $36(23 \%)$ & $4(27 \%)$ & $53(26 \%)$ \\
\hline
\end{tabular}

$A C E$, angiotensin converting enzyme; $A C S$, acute coronary syndrome; $A T 1$, angiotensin type 1; CAD, coronary artery disease; GP, glycoprotein; ISR, in-stent restenosis; MI, myocardial infarction.

${ }^{*} \mathrm{p}<0.001$, ISR $v$ no ISR group; ${ }^{* *} \mathrm{p}<0.05$, progress $v$ no progress group.

angiographically normal segment, or plaque regression, were not found. As a key finding, 12 of a total of $15(80 \%)$ progressive coronary plaques were associated with ISR of the initially treated target lesion, whereas only three plaques showed angiographic progression without concomitant ISR (fig $1 ; \mathrm{p}<0.001$ ). Statistical evaluation revealed smoking $(\mathrm{p}=0.02)$, but no other demographic, angiographic, or procedural variable, associated with progression of untreated plaques (table 1). Baseline CRP concentrations in patients with later coronary progression were significantly increased (18.3 (17.9) mg/l $v 6.9$ (13.1) $\mathrm{mg} / \mathrm{l} ; \mathrm{p}=0.01$ ).

\section{DISCUSSION}

We report on the progression of atherosclerosis in untreated coronary plaques in context with ISR development in a group of patients admitted to a single centre. We have shown that plaque progression after six months occurred in $11 \%$ of preexisting lesions beyond an ISR rate of $28 \%$. As the central finding, lesion progression was highly $(80 \%)$ present with concomitant ISR formation of the target lesion within the same patient. Recent observations had shown 7-12\% progression per year for mild to moderate lesions that were associated with stable angina and conventional medical treatment. Patients with severe coronary artery disease that underwent re-angiography at eight months revealed a progression rate of $11 \%$ with stable angina and 15\% with unstable angina. ${ }^{1}$ Therefore, coronary plaque progression, as observed in the present study, reveals a maximum extent compared to previous findings, indicating a particularly severe and/or active atherosclerosis type present in our patient cohort.

These findings initiate the question for common systemic pathogenic factors that may influence both the treated target lesion and untreated, but diseased coronary plaques. Indeed, our data show elevated pre-procedural CRP concentrations predict ISR and, within the same patients, coincide with the progression of native coronary atherosclerosis. Since CRP exerts chemotactic effects on monocytes and macrophages, ${ }^{2}$ increased leucocyte trafficking post-stent implantation ${ }^{3}$ may include not only circulating monocytes ${ }^{3}$ but also bone marrow derived progenitor cells, that have consistently been found in restenoses. ${ }^{45}$

In conclusion, progression of primarily untreated coronary plaques is strongly associated with target lesion ISR formation. High baseline CRP concentrations may identify patients at risk for both the development of ISR and the progression of coronary atherosclerosis.

\section{Authors' affiliations}

D Skowasch, A Jabs, R Andrié, B Lüderitz, G Bauriedel, Department of Cardiology, Heart Center, University of Bonn, Bonn, Germany

Correspondence to: Dr Dirk Skowasch, Department of Cardiology, Heart Center, University of Bonn, Sigmund-Freud-Str. 25, D-53105 Bonn, Germany; dirk.skowasch@ukb.uni-bonn.de

Accepted 29 June 2004

\section{REFERENCES}

1 Kaski JS, Chester MR, Chen L, et al. Rapid angiographic progression of coronary artery disease in patients with angina pectoris. The role of complex stenosis morphology. Circulation 1995;92:2058-65.

2 Torzewski M, Rist C, Mortensen RF, et al. C-reactive protein in the arterial intima. Role of $C$-reactive protein receptor-dependent monocyte recruitment in atherogenesis. Arterioscler Thromb Vasc Biol 2000;20:2094-9.

3 Fukuda D, Shimada K, Tanaka A, et al. Circulating monocytes and in-stent neointima after coronary stent implantation. J Am Coll Cardiol 2004;43:18-23.

4 Skowasch D, Jabs A, Andrié R, et al. Presence of bone-marrow- and neuralcrest-derived cells in intimal hyperplasia at the time of clinical in-stent restenosis. Cardiovasc Res 2003;60:684-91.

5 Bauriedel G, Jabs A, Skowasch D, et al. Dendritic cells in neointima formation after rat carotid balloon injury. Coordinated expression with anti-apoptotic $\mathrm{Bcl}-2$ and HSP47 in arterial repair. J Am Coll Cardiol 2003;42:930-8. 